\title{
Orbital Motion of Spiral Waves in Excitable Media
}

\author{
V. N. Biktashev \\ Department of Mathematical Sciences, University of Liverpool, Liverpool L69 7ZL, United Kingdom \\ D. Barkley \\ Mathematics Institute, University of Warwick, Coventry CV4 7AL, United Kingdom \\ I. V. Biktasheva \\ Department of Computer Science, University of Liverpool, Liverpool L69 3BX, United Kingdom
}

(Received 31 July 2009; published 3 February 2010)

\begin{abstract}
Spiral waves in active media react to small perturbations as particlelike objects. Here we apply asymptotic theory to the interaction of spiral waves with a localized inhomogeneity, which leads to a novel prediction: drift of the spiral rotation center along circular orbits around the inhomogeneity. The stationary orbits have fixed radii and alternating stability, determined by the properties of the bulk medium and the type of inhomogeneity, while the drift speed along an orbit depends on the strength of the inhomogeneity. Direct numerical simulations confirm the validity and robustness of the theoretical predictions and show that these unexpected effects should be observable in experiment.
\end{abstract}

DOI: 10.1103/PhysRevLett.104.058302

The interest in the dynamics of spiral waves as regimes of self-organization has considerably broadened in the last decades, as they have been found in ever more physical systems of diverse types (magnetic films [1], liquid crystals [2], nonlinear optics [3], new chemical systems [4], and in population [5], tissue [6], and subcellular [7] biology). In a perfectly uniform medium the core of a spiral wave may be anywhere, depending on initial conditions. However, real systems are always heterogeneous, and therefore spiral drift due to inhomogeneity is of great practical interest to applications. Understandably, such drift has been mostly studied in excitable chemical reactions and the heart, where drift due to a gradient of medium properties $[8,9]$ and pinning [10] (anchoring, trapping) to a localized inhomogeneity [11-13] have been observed in experiments and simulations. Interaction with localized inhomogeneity can be considered to be a particular case of the general phenomenon of vortex pinning to material defects [14].

Here we identify a new type of spiral wave dynamics: precession around a localized inhomogeneity along a stable circular orbit. We predict this novel phenomenon theoretically, describe its key features, and confirm it by numerical simulations. We argue that this orbital motion of spiral waves is robust and prevalent, has nontrivial and surprising consequences for applications, and should be directly observable in experiments.

We consider reaction-diffusion equations, which is the most popular class of models describing spiral waves:

$$
\partial_{t} \mathbf{u}=\mathbf{f}(\mathbf{u}, \mathbf{p})+\mathbf{D} \nabla^{2} \mathbf{u},
$$

where $\mathbf{u}, \mathbf{f} \in \mathbb{R}^{\ell}, \mathbf{D} \in \mathbb{R}^{\ell \times \ell}, \mathbf{p} \in \mathbb{R}^{m}, \mathbf{u}(\vec{r}, t)$ is the dynamic vector field, $\vec{r} \in \mathbb{R}^{2}, \mathbf{p}(\vec{r})=\mathbf{p}_{0}+\mathbf{p}_{1}(\vec{r}),\left|\mathbf{p}_{1}\right| \ll 1$, is the vector of parameters, $\mathbf{D}$ is diffusion matrix. For $\mathbf{p}=$
PACS numbers: 82.40.Ck, 05.45.-a, 87.18.Hf, 87.19.Hh

$\mathbf{p}_{0}=$ const, system (1) is assumed to have spiral wave solutions rotating with angular velocity $\omega$ (taken here to be clockwise for $\omega>0$ ),

$$
\mathbf{u}=\mathbf{U}(\rho, \vartheta+\omega t-\Phi)
$$

where $(\rho, \vartheta)$ are polar coordinates defined with respect to the center of rotation $\vec{R}=(X, Y)^{T}$, and $\Phi$ is the initial rotation phase.

In the presence of a small perturbation $\mathbf{p}_{1}(\vec{r}) \neq 0$, the spiral's center of rotation $R=X+i Y$ is not constant but slowly evolves with the equation of motion

$$
\begin{aligned}
\frac{d R}{d t}= & \frac{\omega e^{i \Phi}}{2 \pi} \\
& \times \int_{t-\pi / \omega}^{t+\pi / \omega} e^{-i \omega \tau} \int_{\mathbb{R}^{2}} \int[\mathbf{W}(\rho, \theta)]^{+} \mathbf{h}(\vec{r}, \tau) d^{2} \vec{r} d \tau,
\end{aligned}
$$

where $\rho=\rho(\vec{r}-\vec{R})$ and $\theta=\vartheta(\vec{r}-\vec{R})+\omega \tau-\Phi$ are polar coordinates in the corotating frame of reference, and $\mathbf{h}$ is the perturbation to the right-hand side of Eq. (1). Function $\mathbf{W}$ is called the response function (RF) and defines the sensitivity of the spiral wave position with respect to perturbations in different places. Technically, $\mathbf{W}$ is a projector onto the eigenmode corresponding to the neutral stability with respect to spatial translations and is calculated as the eigenfunction $\mathcal{L}^{+} \mathbf{W}=-i \omega \mathbf{W}$ of the adjoint linearized operator $\mathcal{L}^{+}=\mathbf{D}^{T} \nabla^{2}+\omega \partial_{\theta}+$ $\left(\partial_{\mathbf{u}} \mathbf{f}\left(\mathbf{U} ; \mathbf{p}_{0}\right)\right)^{T}$, see for details [15-17].

For an inhomogeneity $\mathbf{h}=\partial_{\mathbf{p}} \mathbf{f}\left(\mathbf{U}(\rho, \theta) ; \mathbf{p}_{0}\right) \mathbf{p}_{1}(\vec{r})$ uniform inside a disk of radius $R_{i}, \mathbf{p}_{1}(\vec{r})=\frac{\beta}{\pi R_{i}^{2}} H\left(R_{i}-|\vec{r}|\right) \hat{\mathbf{e}}$, $\beta \ll 1$, where $H()$ is the Heaviside step function and $\hat{\mathbf{e}} \in$ $\mathbb{R}^{m},\|\hat{\mathbf{e}}\|=1$, Eq. (3) gives 


$$
\frac{d R}{d t}=-\beta \frac{R}{|R|} F(|R|) .
$$

Here $F$ is the "drift force", defined as the drift velocity per unit inhomogeneity strength $\beta$. For small $R_{i}$, the expression for $F$ simplifies to

$$
F(d)=\int_{0}^{2 \pi} e^{-i \theta}[\mathbf{W}(d, \theta)]^{+} \partial_{\mathbf{p}} \mathbf{f}\left(\mathbf{U}(d, \theta) ; \mathbf{p}_{0}\right) \hat{\mathbf{e}} \frac{d \theta}{2 \pi} .
$$

We calculated the spiral wave solution $\mathbf{U}$ and the response function $\mathbf{W}$ using the method described in $[18,19]$ for the Barkley [20] kinetics $\mathbf{u}=(u, v), \mathbf{p}=(a, b, \boldsymbol{\epsilon}), \mathbf{f}=$ $\left(f_{u}, f_{v}\right)^{T}, \quad f_{u}=\epsilon^{-1} u(1-u)(u-(v+b) / a), \quad f_{v}=$ $u-v, a_{0}=0.7, b_{0}=0.1, \epsilon_{0}=0.02$, and $\mathbf{D}=\left(D_{i j}\right)$, where $D_{11}=1$ and $D_{12}=D_{21}=D_{22}=0$. This model is "excitable", that is, it has a unique spatially uniform steady state, stable with respect to small perturbations [21]. At the chosen parameter values, the spiral wave solutions are stable [22]. Figures 1 (a) and 1(b) show $W_{u}$ and $W_{v}$ components and their location relative to the spiral. Figure 1(c) shows graphs of the radial, $F_{r}(d)=\operatorname{Re}(F(d))$ (positive for attraction) and azimuthal, $F_{a}(d)=\operatorname{Im}(F(d))$ components of the drift force, for the localized inhomogeneity in parameter $b$, i.e., $\hat{\mathbf{e}}=(0,1,0)^{T}$.

The essence of our new finding is that there is the change of sign of radial force $F_{r}(d)$ at $d=d_{1} \approx 3.95$. This follows from the sign changes of $\mathbf{W}$ components as seen in Fig. 1(b). For positive $\beta$, this means attraction to inhomogeneity at small distances and repulsion at larger distances. For negative $\beta$, however, there will be a repulsion from the inhomogeneity at $d<d_{1}$ and attraction at $d>d_{1}$, so that $d=d_{1}$ is a stable distance. The latter corresponds to the drift along an orbit of radius $d_{1}$ with the speed $\left|\beta F_{a}\left(d_{1}\right)\right|$. There is a further root of $F_{r}$ at $d=$ $d_{2} \approx 8.38$; however, the corresponding value of $F_{a}$ is very small, $\sim 10^{-9}$, so no drift is easily observable there.

Figure 2 shows confirmation of the theoretical prediction of the orbital motion by direct numerical simulations (DNS) [19]. Panel (a) illustrates the relationship between the DNS spiral wave solution, its tip and its instantaneous rotation center, and panels (b) and (c) show the center trajectories predicted by the theory and calculated by DNS, for $R_{i}=0.56$ and different values of $\delta_{b}=$ $\beta /\left(\pi R_{i}^{2}\right)$. Trajectories show circular orbits, attracting for $\delta_{b}<0$ and repelling for $\delta_{b}>0$, with the radius indistinguishable from $d_{1}$.

Panels (b) and (c) illustrate two key features of orbital drift: the orbiting speed depends on the strength of the inhomogeneity, while the radius of the orbit does not-it depends only on the properties of the unperturbed medium. These features follow from the theory and are confirmed by DNS: trajectories in panel (c) have the same shape as in panel (b), only the spirals drift along those trajectories faster and in the opposite direction.

Figure 3 provides quantitative comparison between theory and DNS. The theoretical value of the angular velocity of the orbital motion is $\Omega=\left|\delta_{b} \pi R_{i}^{2} F_{a}\left(d_{1}\right) / d_{1}\right|$, implying that $\Omega$ should vary linearly with $\left|\delta_{b}\right|$ with slope $\left|\pi R_{i}^{2} F_{a}\left(d_{1}\right) / d_{1}\right|$. Linearity of $\Omega\left(\delta_{b}\right)$ is indeed found in the DNS, remarkably up until $\left|\delta_{b} / b_{0}\right|=0.9$. The ratio $\Omega / \delta_{b}$ in simulations is slightly smaller than the theoretical value, due to dicretization and approximations used.

Figure 4 compares theory and DNS for the perturbation in the parameter $\epsilon$ rather than $b$. There are now three roots of $F_{r}(d)$, namely, $d_{1} \approx 1.97, d_{2} \approx 3.78$, and $d_{3} \approx 6.45$, two of which are in the experimentally observable range.

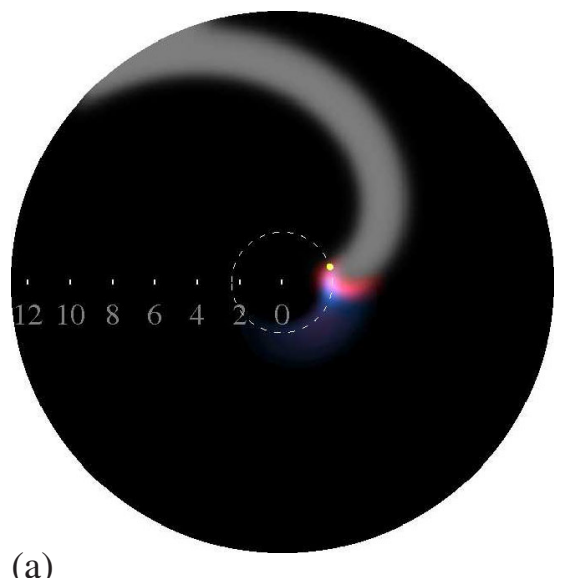

(a)

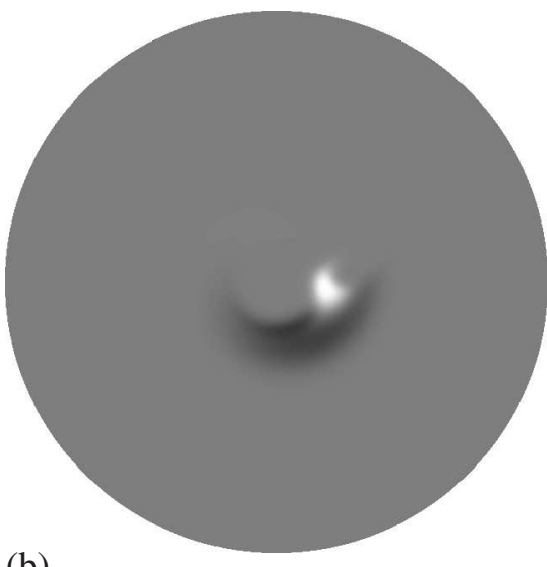

(b)

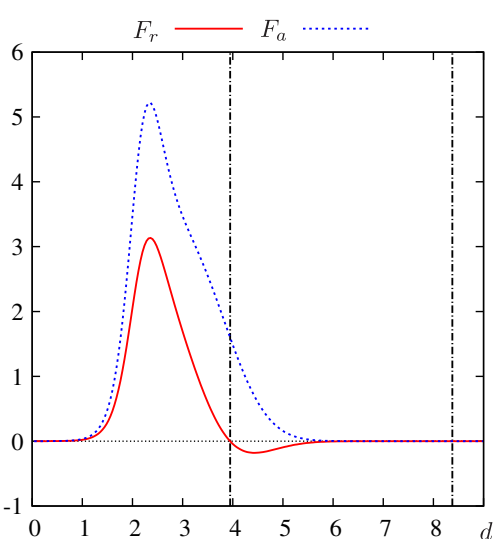

(c)

FIG. 1 (color online). (a) Response function for a spiral wave. The spiral is visualized with a gray-scale plot of the $u$ field. The yellow point indicates the spiral tip and the dashed white line shows its trajectory as the spiral rotates. Superimposed is the response function in term of the $u$ component $\left|W_{u}\right|$ (red) and the $v$ component $\left|W_{v}\right|$ (blue). (b) Enhanced visualization of one component of the response function. $\operatorname{Re}\left(W_{v}\right)$ is plotted with medium gray zero (periphery), light gray positive, and dark gray negative. (c) Drift force. The radial $F_{r}$ (solid red) and azimuthal $F_{a}$ (dashed blue) components of the drift force, as functions of the distance $d$, calculated by (5). The vertical dash-dotted lines show zeros of the radial component, corresponding to the radii at which stationary orbital motion of the spiral wave is possible. 

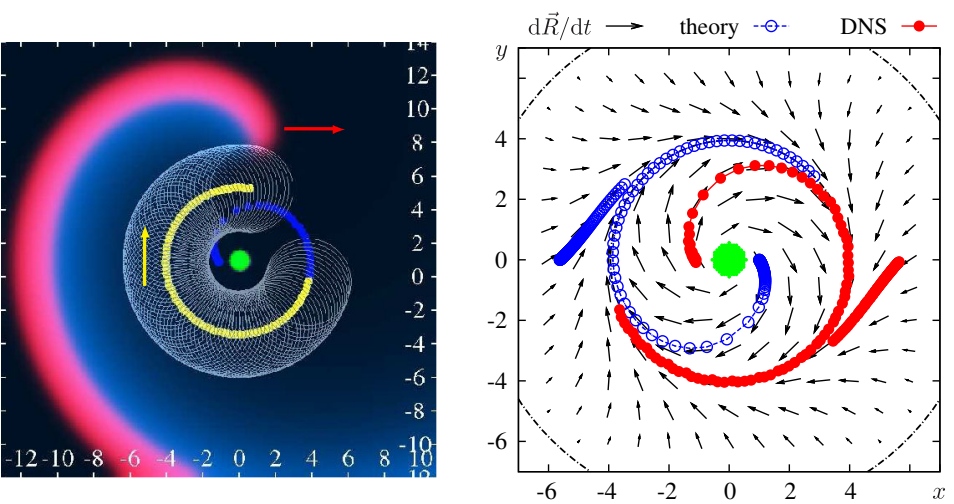

(a) (b)

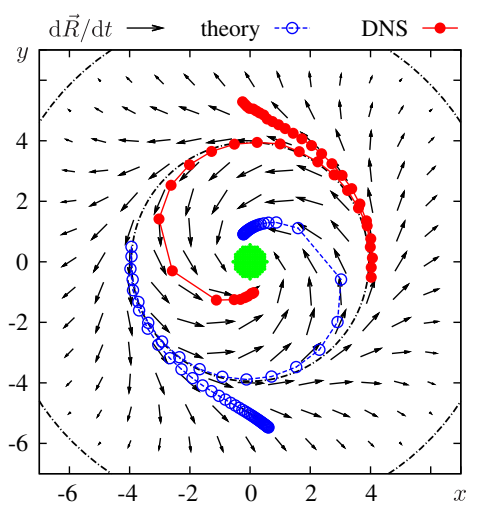

(c)

FIG. 2 (color online). Orbital motion of a spiral. (a) Simulation of a spiral wave in the presence of an inhomogeneity. The spiral starts near the inhomogeneity ( $\delta_{b}=-0.02$, green disk), is repelled from it, and launches into a stable circular clockwise orbit [19]. The $u$ field (red) and $v$ field (blue) of the final spiral are shown. The preceding tip trajectory along the stable orbit is shown by a white line. The preceding centers of rotation $\vec{R}$ are indicated both for this orbit (yellow) and the preceding evolution away from the inhomogeneity (blue). (b) Theoretical vector field (black arrows, nonlinearly scaled for visualization) and predicted trajectories (blue open circles) for the center of a spiral wave near an inhomogeneity (green disk). Actual trajectories for the spiral center from a DNS (red filled circles), with $\delta_{b}=-0.001$. Black dash-dotted circles indicate stationary orbits as predicted by theory. Only every 20th position of the center is shown on both theoretical and DNS trajectories. (c) Same for $\delta_{b}=0.003$.

Roots $d_{1}$ and $d_{2}$ have alternative stability: the $d_{1}$ orbit is unstable for positive $\delta_{\epsilon}$ and stable for negative $\delta_{\epsilon}$, and $d_{2}$ orbit is the other way round. Thus a trajectory starting in between will enter into an orbital motion in any case: to the outer orbit for $\delta_{\epsilon}<0$ and to the inner orbit for $\delta_{\epsilon}>0$; see Figs. 4(b) and 4(c).

In Fig. 4(a), root $d_{1}$ is very close to a root of $F_{a}(d)$, and $F_{a}\left(d_{1}\right)$ is very small. Thus the inner orbit is attracting for $\delta_{\epsilon}>0$ but the motion along it is very slow [Fig. 4(c)] and with short observation time, it may look as if the spiral is attracted to any point at distance $d_{1}$ from the inhomogeneity and stands still there.

To conclude, we have reported a new type of interaction of spiral waves with localized inhomogeneities: orbital motion. This new interaction has key features that should be observable in experiments, namely, the orbiting speed depends on the strength of the inhomogeneity, while the stationary orbit radii form a discrete set depending only on the properties of the unperturbed medium. The phenomenon is rather generic: we have found it in a number of other models and for more general shape of inhomogeneity [17]. The possibility of orbital drift, related to a change of sign of an equivalent of $F_{r}(d)$ was discussed at a speculative level in [23]; how often this phenomenon may occur in reality is a more complicated question. The equivalent of response functions calculated in [24] has a structure which suggests that for large-core spirals there is an infinite set of orbits. In practice, orbital motion can only be observed for lower orbits where the orbiting speed is noticeable. The orbits have alternating stability, depending on the sign of the inhomogeneity. From this viewpoint, pinning as considered in [25] in the same model as here, appears as a degenerate case of orbital motion, with a zero radius.
In certain circumstances, while an orbiting spiral may have the same macroscopic signature as a meandering one [26], the microscopic details leading to this motion are different. Meander, in the proper sense, is due to internal instabilities of a spiral wave, whereas orbital motion is due to inhomogeneity. E.g., in orbiting, the "meandering pattern" determined by $\Omega / \omega$ will change depending on the inhomogeneity strength.

In heart muscle, pinning of reentrant waves of excitation has been identified as a mechanism of conversion of ventricular fibrillation into ventricular tachycardia [27] and shown to interfere with antitachycardia pacing [28]. Orbital motion is fundamentally more general than classical pinning: in pinning, the spiral is attracted at all distances to the inhomogeneity of a certain sign, while orbital motion occurs when repulsion at small distances changes to attraction at larger distances, and this can happen at various distances and at either sign of inhomogeneity. The important practical consequence of the orbital motion is

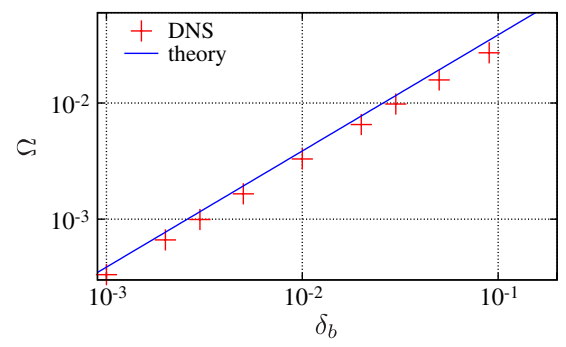

FIG. 3 (color online). Angular speed $\Omega$ of orbital motion of the spiral wave as a function of the amplitude of the parametric inhomogeneity $\delta_{b}$ : theoretical prediction vs measurements from direct numerical simulations. 


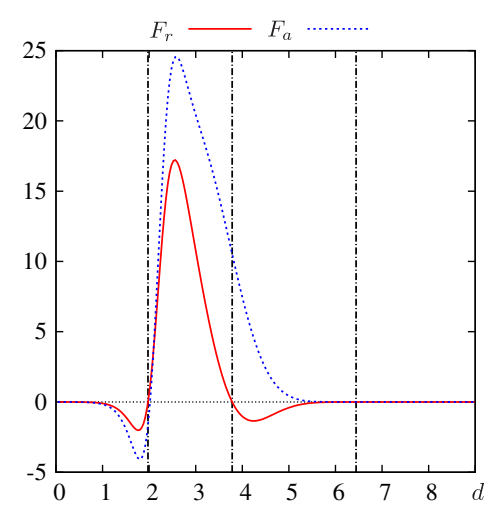

(a)

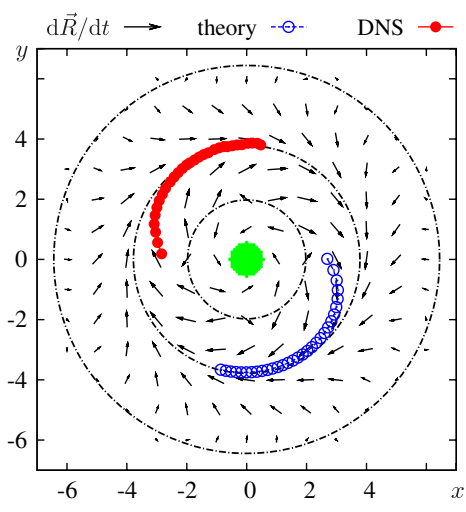

(b)

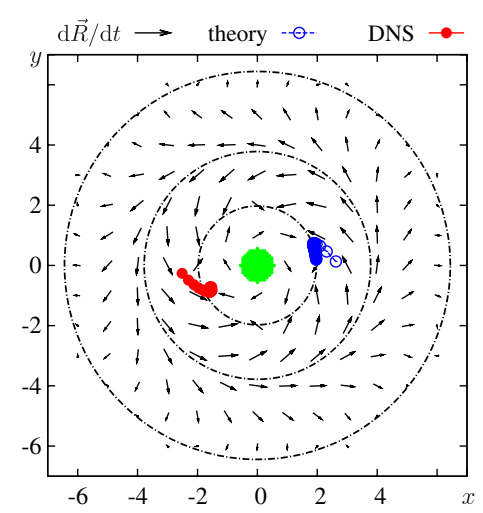

(c)

FIG. 4 (color online). Orbital motion of the spiral due to perturbation in parameter $\epsilon$. (a) Drift force components as functions of distance for this inhomogeneity. The notation is the same as in Fig. 1(c). (b),(c) Comparison of theoretical predictions and simulations, for (b) $\delta_{\epsilon}=-0.001$ and (c) $\delta_{\epsilon}=0.001$. The notation is the same as in Figs. 2(b) and 2(c). Shown are pieces of trajectories of the same temporal length $t=0 \ldots 500$.

that a spiral may be bound to inhomogeneities of either sign, even if it is repelled from the inhomogeneity at small distances.

V. N. B. is grateful to V. I. Krinsky for inspiring discussions regarding the problem of pinning. This study has been supported in part by EPSRC grants EP/D074789/1 and EP/D074746/1.

[1] A. G. Shagalov, Phys. Lett. A 235, 643 (1997).

[2] P. Oswald and A. Dequidt, Phys. Rev. E 77, 051706 (2008).

[3] M. Le Berre, E. Ressayre, A. Tallet, and M. Tlidi, Phys. Rev. E 71, 036224 (2005).

[4] K. Agladze and O. Steinbock, J. Phys. Chem. A 104, 9816 (2000).

[5] O. A. Igoshin, R. Welch, D. Kaiser, and G. Oster, Proc. Natl. Acad. Sci. U.S.A. 101, 4256 (2004).

[6] M. A. Dahlem and S.C. Müller, Biol. Cybern. 88, 419 (2003).

[7] T. Bretschneider et al., Biophys. J. 96, 2888 (2009).

[8] V.G. Fast and A.M. Pertsov, J. Cardiovasc. Electrophysiol. 3, 255 (1992).

[9] C. Luengviriya, U. Storb, M. J.B. Hauser, and S.C. Müller, Phys. Chem. Chem. Phys. 8, 1425 (2006).

[10] Note that the term "pinning" can also be used in a completely different sense, see O. Jensen, V.O. Pannbacker, E. Mosekilde, G. Dewel, and P. Borckmans, Phys. Rev. E 50, 736 (1994), although "self-pinning" perhaps would be more accurate in that case.

[11] S. Nettesheim, A. von Oertzen, H. H. Rotermund, and G. Ertl, J. Chem. Phys. 98, 9977 (1993).

[12] A. M. Pertsov, J. M. Davidenko, R. Salomonsz, W. T. Baxter, and J. Jalife, Circ. Res. 72, 631 (1993).
[13] Z. Y. Lim, B. Maskara, F. Aguel, R. Emokpae, and L. Tung, Circulation 114, 2113 (2006).

[14] S. Lugomer, Y. Fukumoto, B. Farkas, T. Szörényi, and A. Toth, Phys. Rev. E 76, 016305 (2007).

[15] V. N. Biktashev and A. V. Holden, Chaos Solitons Fractals 5, 575 (1995).

[16] I. V. Biktasheva and V. N. Biktashev, Phys. Rev. E 67, 026221 (2003).

[17] I. V. Biktasheva, D. Barkley, V. N. Biktashev, and A.J. Foulkes, Computation of the drift velocity of spiral waves using response functions (to be published).

[18] I. V. Biktasheva, D. Barkley, V. N. Biktashev, G. V. Bordyugov, and A.J. Foulkes, Phys. Rev. E 79, 056702 (2009).

[19] See supplementary material at http://link.aps.org/ supplemental/10.1103/PhysRevLett.104.058302 for a movie and details of numerical procedures.

[20] D. Barkley, Physica (Amsterdam) 49D, 61 (1991).

[21] M. Kness, L. S. Tuckerman, and D. Barkley, Phys. Rev. A 46, 5054 (1992).

[22] D. Barkley, Phys. Rev. Lett. 72, 164 (1994).

[23] V. G. LeBlanc and C. Wulff, J. Nonlinear Sci. 10, 569 (2000).

[24] Y.E. Elkin and V. N. Biktashev, J. Biol. Phys. 25, 129 (1999).

[25] D. Pazó, L. Kramer, A. Pumir, S. Kanani, I. Efimov, and V. Krinsky, Phys. Rev. Lett. 93, 168303 (2004).

[26] Orbiting may in fact have been observed by Zou et al., Phys. Rev E 47, R800 (1993); see p. R802, however, it is difficult to be certain as no details were given.

[27] M. Valderrabano, Y. H. Kim, M. Yashima, T. J. Wu, H. S. Karagueuzian, and P. S. Chen, J. Am. College Cardiol. 36, 2000 (2000).

[28] C. M. Ripplinger, V. I. Krinsky, V. P. Nikolski, and I. R. Efimov, Am. J. Physiol. 291, H184 (2006). 